\title{
Expression of mucins (MUC1, MUC2, MUC3, MUC4, MUC5AC and MUC6) and their prognostic significance in human breast cancer
}

\author{
Emad A Rakha ${ }^{1}$, Richard WG Boyce ${ }^{1}$, Dalia Abd El-Rehim ${ }^{1}$, Thomas Kurien ${ }^{1}$, \\ Andrew R Green ${ }^{1}$, Emma C Paish ${ }^{1}$, John FR Robertson ${ }^{2}$ and Ian O Ellis ${ }^{1}$ \\ ${ }^{1}$ Department of Histopathology, The Breast Unit, Nottingham City Hospital, University of Nottingham, \\ Nottingham, UK and ${ }^{2}$ Department of Surgery, Nottingham City Hospital, University of Nottingham, \\ Nottingham, UK
}

\begin{abstract}
Mucins are a large family of glycoproteins expressed by many epithelial cells and their malignant counterparts. Much interest has been focused on expression of its members in breast cancer because of their potential role as prognostic indicators and their involvement in cancer therapy. We have examined 1447 cases of invasive breast carcinoma with a long-term follow-up, using tissue microarray (TMA) technology and immunohistochemistry to evaluate the expression profiles of several mucins (MUC1, MUC2, MUC3, MUC4, MUC5AC and MUC6) and to assess their prognostic value. We detected MUC1 expression in $91 \%$ of tumours. MUC1 overexpression was associated with a lower grade, smaller tumour size, a higher oestrogen receptor (ER)-positive phenotype and absence of both regional recurrence and distance metastasis. The subcellular localization but not the level of expression had a prognostic value in predicting outcome. The aberrant cytoplasmic and membranous localization of MUC1 was associated with poor outcome compared with apical localization, which is the normal physiological site of expression. MUC2 expression was noticed in only $8.3 \%$ of all cases and was restricted to the cytoplasm of the tumour cells. An inverse trend was identified between MUC2 expression and lymph node stage and vascular invasion status. On excluding cases of mucinous carcinoma from the analysis, the inverse association with vascular invasion was still defined and in addition an inverse association with ER status emerged. MUC3 expression was detected in $91 \%$ of cases and its expression was associated with increased local recurrence, and lymph node stage. The membranous expression of MUC3 was found to be a potentially poor prognostic feature, with higher grade and poorer Nottingham Prognostic Index (NPI), and negative ER expression. MUC4, MUC5AC and MUC6 were expressed in 95,37 and $20 \%$ of cases, respectively. Apart from an association between MUC4 expression and tumour grade and between MUC6 and ER-negative tumours, no other associations with any clinicopathological variables were found. Apart from the higher expression of MUC2 and MUC6 in mucinous carcinomas, no association was found between the expression of different mucins and tumour type. No association between the level of expression of any of the studied mucins and patient outcomes has been identified. In conclusion, most breast carcinomas express MUC1, MUC3 and MUC4. Among the various mucins expressed in breast cancer, MUC1 and MUC3 are potential prognostic indicators, MUC1 having the strongest relationship with patient outcome.

Modern Pathology (2005) 18, 1295-1304. doi:10.1038/modpathol.3800445; published online 24 June 2005
\end{abstract}

Keywords: breast cancer; mucin; immunohistochemistry; prognosis; tissue microarray

Much effort is being carried out to identify markers that have biological and therapeutic significance in

Correspondence: Dr EA Rakha, BM, BCh, MSc, PhD, Department of Histopathology, Sandringham Building, Leicester Royal Infirmary, University Hospitals of Leicester, Leicester LE1 5WW, UK.

E-mails: emadrakha@yahoo.com, mrxeaaer@nottingham.ac.uk

Received 24 March 2005; accepted 1 April 2005; published online 24 June 2005 breast cancer. Mucins are a large family of glycoproteins expressed by various epithelial cell types and malignant counterparts. They are classified into two main classes: membrane bound including MUC1, MUC3A, MUC3B, MUC4, MUC12, MUC13 and MUC17, and secreted or gel forming including MUC2, MUC5AC, MUC5B and MUC6. ${ }^{1}$ All share common structural features but are distinct in their tandem repeat peptides. ${ }^{2}$ Abnormal expression of mucins has been reported to accompany cancer 
development, influence cellular growth, differentiation, transformation, adhesion, invasion and immune surveillance. ${ }^{3}$ Numerous studies showed the overexpression of MUC1 in breast cancer, along with other cancers such as those of the colon and pancreas; ${ }^{4}$ however, studies of the expression of other mucins in breast cancer are more limited.

MUC1 is involved with cell signalling, immunoregulation and inhibition of cell-cell and cellmatrix adhesion. ${ }^{5}$ MUC1 cytoplasmic domain has been observed to compete for and interact with $\beta$ catenin through a similar motif found in E-cadherin, thus inhibiting the formation of E-cadherin$\beta$-catenin complex. ${ }^{6}$ This finding has further been confirmed by a subsequent in vitro study on breast cancer cell line. ${ }^{7,8}$ Therefore, MUC1 may participate in tumour cell detachment, invasion and metastases and therefore, associated with aggressive tumour behaviour and poor prognosis. The cytoplasmic domain has an additional role where phosphorylated MUC1 binds to Grb2/SOS, signalling mediators of tyrosine kinase receptors. ${ }^{9}$ In addition, colocalization and interactions between MUC 1 and members of EGFR family members have also been reported. ${ }^{10}$ MUC1 mucin is normally present on the apical surface of secretory epithelia; however, in the malignant tissues, its expression is usually variable in amount and cellular localization. ${ }^{11}$ The high level of the aberrant MUC1 expression in breast cancer and other cancers results in antigenically recognizable epitopes on the MUC1 molecule leading to stimulation of the immune response and making MUC1 a potential immunotherapeutic target. $^{12}$

MUC2, MUC5AC and MUC6 are key proteins for producing the mucus that protects and lubricates epithelial surfaces. MUC2 is the major secretory glycoprotein expressed abundantly by intestinal and airway epithelium. ${ }^{13}$ Its expression is a common feature of all mucinous carcinomas derived from different organs including breast, colon and prostate where it may act as a potential prognostic indicator. ${ }^{14-16}$ MUC2 is expressed in mucinous breast cancer and may play a role in prevention of tumour invasion. ${ }^{17}$ Its expression is associated with aggressive tumour behaviour in other types of invasive ${ }^{18}$ and intraductal carcinomas of the breast. ${ }^{19}$

MUC5AC is found mainly in the mucosal layer of the cardia, fundus and antrum of the stomach whereas MUC6 is located in the pyloric glands, ${ }^{20,21}$ both have similar properties in protecting epithelia and both are generally not detected in normal breast tissues. ${ }^{22}$ Matsukita et $a l^{23}$ have shown a correlation between MUC6 expression and mucinous carcinoma of the breast. Previous studies have shown no association between MUC5AC or MUC6 and tumour size, histological grade, node status or oestrogen receptor (ER) status. ${ }^{22}$

MUC3 is found in the intestine and consists of two distinct genes MUC3A and MUC3B. It has been shown that MUC3 is upregulated as a result of steroids in vitro, ${ }^{24}$ leading to the potential association of aberrant hormonal mechanisms and a loss of controlled expression of mucin genes in breast cancer. In gastric carcinoma, MUC3 expression has been associated with poor prognosis. ${ }^{25}$

MUC4 normally provides a protective layer of mucus to the epithelial cell of the breast. The antiadhesive antirecognition barrier provided by MUC4 expression may potentially be used as a mechanism of preventing apoptosis and indirectly increasing tumour proliferation. ${ }^{26}$ It has also been shown to be a modulator of the c-erbB-2 receptor tyrosine kinase via two epithelial growth factor-like domains contained within the transmembrane portion of the complex (ASGP-2) ${ }^{26,27}$ Overexpression of MUC4 has been shown to block cell-cell and cellmatrix interactions, protect tumour cells from immune surveillance and promote metastasis. In addition, as a ligand for ErbB2, MUC4 can potentiate phosphorylation of ErbB2 and potentially alter signals generated from this receptor. ${ }^{28}$ The deregulation of MUC4 may be a factor for tumour progression and its altered expression has been found in a number of carcinomas. ${ }^{29,30}$

In this study, we have assessed the expression profiles of different mucins and evaluate their prognostic significance in a large and well-characterized series of breast cancer, prepared as tissue microarrays (TMA), using immunohistochemistry.

\section{Materials and methods}

\section{Patients and Tumours}

A consecutive series of 1447 cases of invasive breast carcinoma available from the Nottingham Tenovus Primary Breast Carcinoma Series were used. This is a well-characterized series of primary operable invasive breast cancer that has been previously used to study a wide range of proteins. ${ }^{31-33}$ The patients had a median age of 54 years (range 18-70 years). The mean follow-up time was 82 months (range 7-138 months). Patients' clinical history and cancer characteristics including tumour type ${ }^{34}$ histological grade, ${ }^{35}$ tumour size, lymph node (LN) status, Nottingham Prognostic Index (NPI), ${ }^{36,37}$ ER expression and survival data including survival time (OS), disease-free interval (DFI), recurrence and development of distant metastasis were available for all patients.

\section{TMA and Immunohistochemistry}

TMA were prepared as previously described. ${ }^{38}$ In brief, tissue $0.6 \mathrm{~mm}$ diameter cores were punched from representative tumour regions of each donor block and arrayed into a new recipient paraffin blocks using a tissue microarrayer (Beecher Instruments, Sun Prairie, WI, USA). These TMA blocks were constructed in triplicate, each containing one 
sample from a different region of the tumour. TMA sections, $4 \mu \mathrm{m}$, were stained immunohistochemically using the standard streptavidin-biotin complex method as previously described. ${ }^{16,31,39}$ Briefly, tissue slides were deparaffinized with xylene and then rehydrated through three changes of alcohol. Endogenous peroxidase activity was blocked by incubation in a $0.3 \%$ hydrogen peroxide/methanol buffer. Antigen retrieval was carried out by microwave treatment of the slides in sodium citrate buffer ( $\mathrm{pH}$ 6.0) for $20 \mathrm{~min}$. The slides were rinsed in trisbuffered saline (TBS) (pH 7.6) and incubated with normal swine serum (NSS) in TBS (1:5) to block nonspecific staining. The slides were incubated for $1 \mathrm{~h}$ with the corresponding anti-mucin antibody (Table 1). After washing with TBS, sections were incubated with the secondary antibody (biotinylated goat anti-mouse/rabbit immunoglobulin; Duet K 0492, DakoCytomation, Denmark) (1:100) for $30 \mathrm{~min}$ followed by the avidin-biotin complex (1:100) for a further $45 \mathrm{~min}$. 3-3'Diaminobenzidine tetrahydrochloride (Dako liquid DAB plus, K3468, Denmark) was used as a chromogen with the addition of copper sulphate to enhance staining. All sections were counterstained with Mayer's haematoxylin. Positive and negative control slides (according to the manufacturer's data sheet of each antibody) were included in every experiment in addition to the internal positive controls (normal breast tissue).

\section{Evaluation of Immunohistochemical Staining}

For assessment of staining, the modified histochemical score (H-score) ${ }^{40}$ was used, which includes an assessment of both the intensity of staining and the percentage of stained cells. For the intensity, a score index of $0,1,2$ and 3 corresponding to negative, weak, moderate and strong staining intensity was used and the percentage of positive cells at each intensity was estimated subjectively. In cases of MUC2, which was expressed in a small proportion of cases, positivity was defined by detection of positive expression in $>5 \%$ of the malignant cells. ${ }^{22}$ With other mucin antibodies, positivity was defined by detection of positive expression in $>5 \%$ of the malignant cells and positive expression was stratified into two groups below and above the median of

Table 1 Mucin antibodies

\begin{tabular}{llll}
\hline Mucin & Manufacturer & Clone & Dilution \\
\hline NCL-MUC1 & Novocastra & Ma695 & $1: 300$ \\
NCL-MUC1-core & Novocastra & Ma552 & $1: 250$ \\
MUC2 & Novocastra & Ccp58 & $1: 250$ \\
MUC3 & NeoMarkers & 1143/B7 & $1: 100$ \\
MUC4 & ZYMED & 1G8 & $1: 10$ \\
MUC5AC & Novocastra & CLH2 & $1: 25$ \\
MUC6 & Novocastra & CLH5 & $1: 25$ \\
\hline
\end{tabular}

expression corresponding to low and high expression. ${ }^{23}$ In case of MUC1, additional assessment of the subcellular localization was conducted. The pattern of expression was as follows: luminal/ apical, combined luminal and cytoplasmic and cytoplasmic with circumferential membranous expression. Two cores were evaluated from each tumour. Each core was scored individually then the mean of the two readings was calculated. The cases were scored by one observer without previous knowledge of the outcomes on two separate occasions.

\section{Statistical Analysis}

Associations between clinico-pathological variables and expression of different mucin antigens were analysed using the $\chi^{2}$-test. Correlation between protein expression levels and overall survival (OS) and -DFI was analysed using Kaplan-Meier curves with the differences estimated using the Mantel-Cox log-rank test. A $P$-value $<0.05$ was considered significant.

\section{Results}

\section{MUC1 Expression and Relation to Clinico-Pathological Features}

The expression of MUC1 was assessed using two monoclonal antibodies (NCL-MUC1-core and NCLMUC1). NCL-MUC1-core recognizes MUC1 mucin core peptide (TRPAPG) (nonglycosylated MUC1). NCL-MUC1 recognizes a carbohydrate epitope of the MUC1-glycoprotein (data sheet from Novocastra Laboratories Ltd). For the two antibodies used, a highly significant correlation was detected (correlation coefficient $=0.733, P<0.001$ ) between the expressions of both markers. Therefore, we considered the expression of one marker only (NCL-MUC1 (clone Ma695)). Positive MUC1 expression was found in the majority of the tumours $(91 \%)$ of which $42 \%$ showed positive expression level above the median (H-score $=200$ ) (high expression).

MUC1 expression was inversely associated with tumour histological grade and tumour size where MUC1-negative phenotype was more frequently noticed in higher grade and in tumours $>1.5 \mathrm{~cm}$ compared with the smaller ones. There was an association between MUC1 expression and regional recurrence and distant metastasis where negative expression of MUC1 was noticed in more tumours with positive regional recurrence $\left(\chi^{2}=10.4, \mathrm{df}=2\right.$, $P=0.006)$ and or those with positive distant metastasis $\left(\chi^{2}=6.47, \mathrm{df}=2, P=0.03\right)$ and an association of borderline significance with the presence of LN metastasis $\left(\chi^{2}=5.1, \mathrm{df}=2, P=0.08\right)$. An association was detected between MUC1 expression and ER status where high MUC1 overexpressing tumours 
were more frequently ER positive (47.1 vs $32.2 \%$ ) (Table 2).

\section{Subcellular Localization in Relation to Clinico-Pathological Features}

There was marked heterogeneity regarding MUC1 subcellular localization (Figures 3a-c). The majority of tumours showed cytoplasmic expression with circumferential membranous accentuation while exclusive luminal/apical expression was seen only in $4.5 \%$ of tumours (Table 3). There was an association between subcellular localization and tumour grade. Cytoplasmic and membranous expression was more frequently associated with grade 3 tumours while luminal and combined luminal and cytoplasmic expression were more frequently associated with lower tumour grade $\left(\chi^{2}=354.73, \mathrm{df}=6\right.$, $P<0.001)$. Regarding ER status, we found that cytoplasmic and membranous expression was significantly associated with the ER-negative phenotype compared with luminal or combined luminal and cytoplasmic expression $\left(\chi^{2}=103.96, \mathrm{df}=3\right.$, $P<0.001)$. The luminal expression was associated with absence of vascular invasion $\left(\chi^{2}=8.9, \mathrm{df}=3\right.$, $P=0.031), \mathrm{LN}$ metastasis $\left(\chi^{2}=9.6, \mathrm{df}=3, P=0.022\right)$ and distant metastasis $\left(\chi^{2}=24.72, \mathrm{df}=3, P<0.001\right)$. In addition, the subcellular localization was associated with the histological tumour type $\left(\chi^{2}=603.59, \mathrm{df}=45, P<0.001\right)$,

Table 2 Correlation between MUC1 expression and clinicopathological variables

\begin{tabular}{|c|c|c|c|c|c|c|}
\hline \multirow[t]{2}{*}{ Variables } & \multicolumn{4}{|c|}{ MUC1 expression } & \multirow[t]{2}{*}{$\chi^{2}$} & \multirow[t]{2}{*}{ P-value } \\
\hline & Total & Negative & Low & High & & \\
\hline \multicolumn{7}{|l|}{ Grade } \\
\hline 1 & 242 & 7 (2.9) & $123(50.8)$ & $112(46.3)$ & 68.37 & $<0.001$ \\
\hline 2 & 477 & $24(5.0)$ & $204(42.8)$ & $249(52.2)$ & & \\
\hline 3 & 728 & 109 (15.0) & $365(50.1)$ & $254(34.9)$ & & \\
\hline \multicolumn{7}{|l|}{ Size $(\mathrm{cm})$} \\
\hline$\leq 1.5$ & 479 & 33 (6.9) & $232(48.4)$ & $214(44.7)$ & 6.55 & 0.038 \\
\hline$>1.5$ & 968 & $107(11.1)$ & $459(47.4)$ & $402(41.5)$ & & \\
\hline \multicolumn{7}{|c|}{ 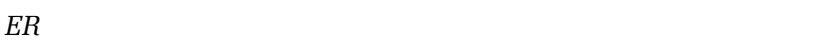 } \\
\hline Negative & 435 & $78(17.9)$ & 217 (49.9) & $140(32.2)$ & 61.47 & $<0.001$ \\
\hline Positive & 1002 & $60(6.0)$ & $470(46.9)$ & $472(47.1)$ & & \\
\hline
\end{tabular}

$\mathrm{ER}=$ oestrogen receptor.

Table 3 Subcellular localization of MUC1 expression

\begin{tabular}{lrr}
\hline Site of expression & No. of cases & $\%$ \\
\hline Pure luminal/apical & 60 & 4.5 \\
Luminal and cytoplasmic & 245 & 18.4 \\
Cytoplasmic with circumferential & 1028 & 77.1 \\
membranous accentuation & & \\
\hline
\end{tabular}

\section{Survival Analyses}

Kaplan-Meier survival analyses revealed no significant association between the level MUC1 expression and either OS or DFI $(P=0.4791$ and 0.6053, respectively) in general. There also was no association between the level of expression and outcome on comparing the level of expression in tumours showing cytoplasmic, membranous or apical expression in particular. However, MUC1 subcellular localization showed differences in relation to both OS and DFI $(P<0.001)$ where tumours with cytoplasmic and membranous pattern of expression have poorer OS and shorter DFI compared with those with pure luminal expression (Figures 1 and 2).

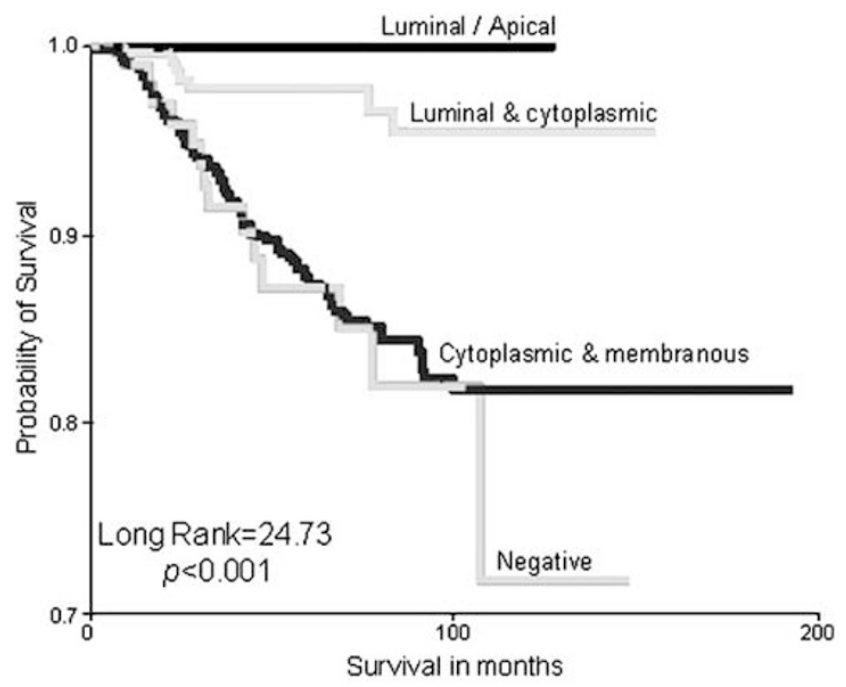

Figure 1 Correlation between the subcellular distribution of MUC1 expression and overall survival (Kaplan-Meier method and log-rank test).

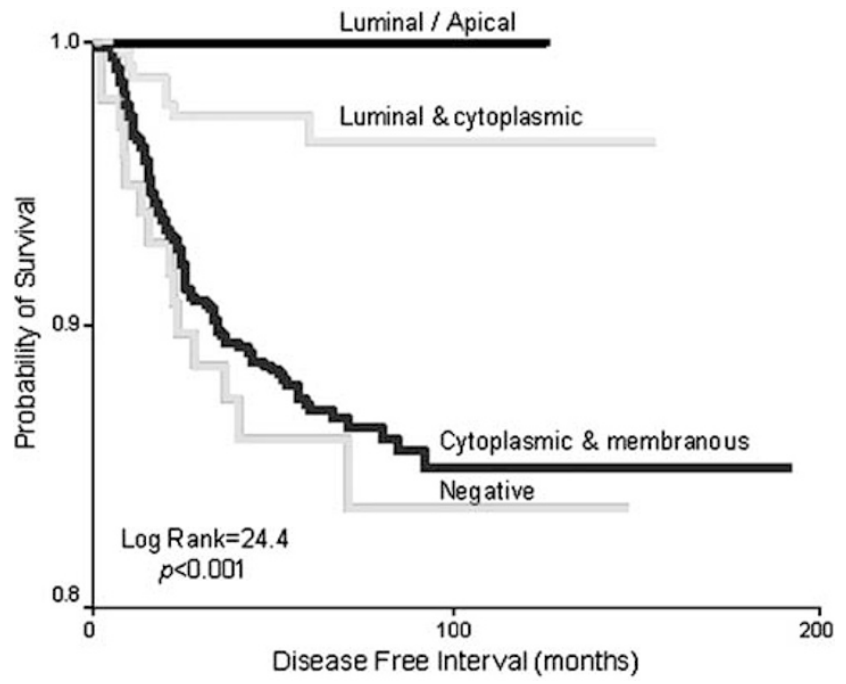

Figure 2 Correlation between the subcellular distribution of MUC1 expression and disease-free interval. 


\section{MUC2 Expression}

Only $8.3 \%$ of the cases showed MUC2 expression, which was restricted to the cytoplasm of the tumour cells. There was high expression rates of MUC2 in mucinous carcinomas 13/16 (81.3\%) in contrast with the significantly lower expression rates seen with other tumour types (Figures $3 \mathrm{~d}$ and e).

MUC2 expression showed an inverse association with both LN stage $(P=0.034)$ and vascular invasion $(P=0.024)$ (Table 4$)$. In nonmucinous tumour types $(n=104)$, MUC2 expression was inversely associated with vascular invasion $\left(\chi^{2}=4.13, P<0.042\right)$ where MUC2-expressing tumours were less frequently showing vascular invasion. A significant inverse association was also noticed between positive MUC2 expression and ER status $\left(\chi^{2}=4.93\right.$, $P<0.026)$. There were no further associations between MUC2 expression and any other clinicopathological features. Kaplan-Meier survival analyses revealed no prognostic significance of MUC2 expression in breast cancer.

\section{MUC3 Expression}

MUC3 expression was detected in the cytoplasm of the malignant cells in $91 \%$ of cases; of these, $21 \%$ also showed membranous staining (Figure 3f). There was an association between MUC3 expression and LN stage $\left(\chi^{2}=9.04, P=0.02\right)$, development of local recurrences $\left(\chi^{2}=10.25, P=0.017\right)$ and of borderline significance with regional recurrence $\left(\chi^{2}=7.6\right.$, $P=0.05)$. An association was also found between its membranous expression and increased grade $\left(\chi^{2}=134.87, \quad P<0.001\right), \quad$ a poorer NPI group $\left(\chi^{2}=30.98, \quad P<0.001\right), \quad$ loss of $\mathrm{ER} \quad\left(\chi^{2}=24.33\right.$, $P<0.001)$ and presence of vascular invasion $\left(\chi^{2}=4.1, P=0.04\right)$.

\section{MUC4 and MUC5AC Expression}

MUC4 and MUC5AC were expressed in 95 and 37\% of the studied cases, respectively (Figures $3 g$ and $h$ ). Apart from an association between MUC4 expression and tumour grade $\left(\chi^{2}=10.7, P=0.02\right)$ and that of borderline significance between MUC5AC expression and menopausal status $\left(\chi^{2}=5.5, P=0.06\right)$, there were no further associations with other clinicopathological variables.

\section{MUC6 Expression}

Positive expression of MUC6 was detected in 20\% of cases (Figure 3i). There was high expression rates of MUC6 in mucinous carcinomas in contrast with other tumour types. Apart from an association between its expression and tumours negative for ER receptors $\left(\chi^{2}=9.1, P=0.01\right)$, no association was found with the other studied parameters. MUC6 expression appeared to be good survival factor (Kaplan-Meier survival analysis, $P=0.053$ ). However, Cox multivariate analysis showed that MUC6 was not independently responsible for an increase in survival rate.

\section{Discussion}

This study has investigated the expression profiles and prognostic value of different mucins in breast cancer cases using TMA technology. In our series, MUC1 was expressed in the majority of breast cancer cases using two different antibodies; one directed against the carbohydrate side chain (MUC1) and the other directed against the apomucin protein backbone (MUC1-core). This is in concordance with previous studies using different antibodies directed against tandem repeat peptides, ${ }^{11}$ carbohydrate side chain ${ }^{41}$ of the extracellular domain or against the cytoplasmic domain. ${ }^{42}$ We found associations between MUC1 expression and tumour grade and ER status where such relationships have previously been reported indicating high correlation between MUC1 expression and functional differentiation of the tumour cells. ${ }^{11,30,43-45}$

The presence of apical cellular localization is an indicator of intact MUC1 pathway that is associated with functional differentiation and good prognosis while the presence of other aberrant patterns of expression that are commonly seen in breast cancer is an indicator of defective MUC1 pathway that is associated with lack of functional differentiation and worse prognosis. ${ }^{11}$ In this study, the expression of MUC1 alone was not predictive of the patients outcome, however, there was an association between its subcellular localization and OS where cytoplasmic and/or membranous expression was associated with poorer survival compared with apical and combined apical and cytoplasmic localization. The subcellular localization of MUC1, but not the expression level, has been previously reported to have a prognostic value in predicting outcome in breast cancer using TMA technology. ${ }^{46}$ The associations between cytoplasmic expression and poor outcome and between apical expression and favourable outcome have also, been reported. ${ }^{47,48}$ Other studies, however, found no association between either level of expression or the site of expression and outcome. ${ }^{49-51}$

In our series, MUC2 was expressed with a very high frequency in mucinous carcinomas compared with other tumour types as previously reported. ${ }^{23,30,52}$ A previous study has described MUC2 expression in the adjacent in situ lesions as well as adjacent and remote normal breast tissue and suggested that altered MUC2 expression pattern existing in normal breast tissue is a directional vector in histogenesis of such histological tumour type. ${ }^{52}$ However, in this study, we could not confirm 

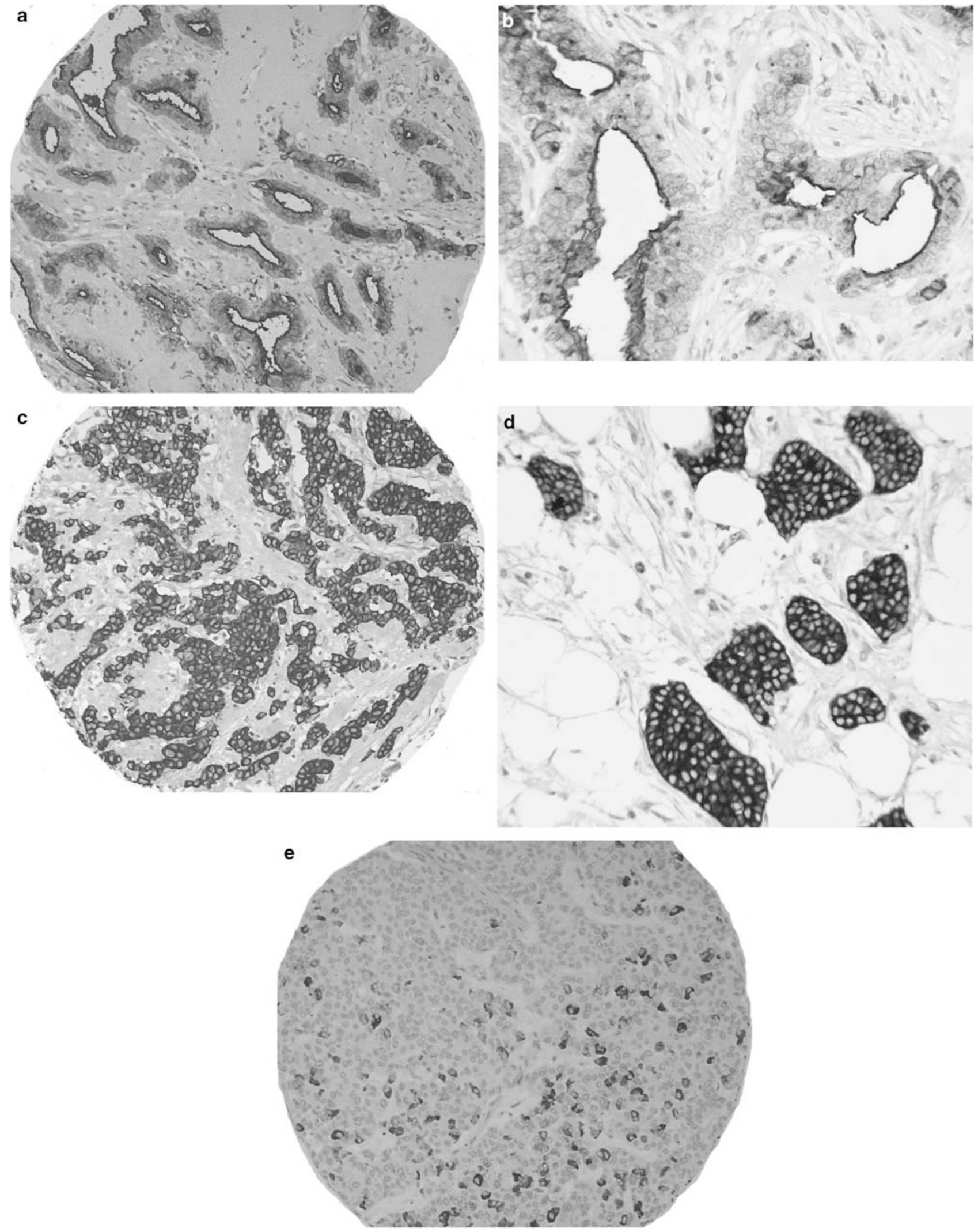

Figure 3 Immunohistochemistry for mucins in breast carcinomas. (a and b) A case of low-grade ductal no special type (NST) carcinoma showing luminal/apical expression of MUC1. (c) A case of ductal/NST carcinoma showing strong cytoplasmic and luminal expression of MUC1. (d) A case of mucinous carcinoma showing strong cytoplasmic expression of MUC2. (e) A case of ductal/NST carcinoma showing scattered positive cytoplasmic expression of MUC2. (f) A case of ductal/NST carcinoma showing positive expression of MUC3. (g-i) Positive expression of MUC 4 (g), MUC5AC (h) and MUC6 (i) in breast carcinoma. 

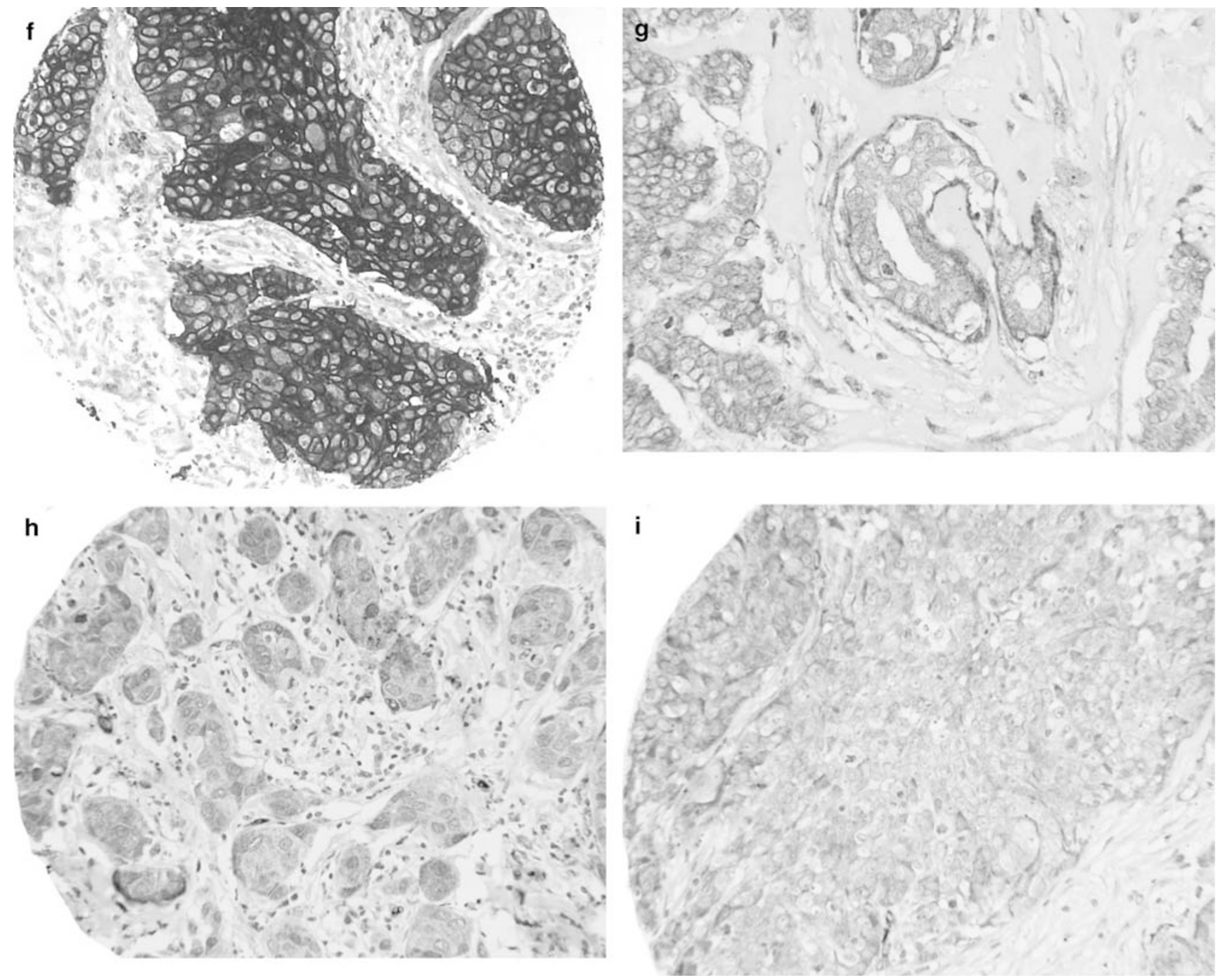

Figure 3 Continued.

Table 4 Correlation between MUC2 expression and clinicopathological variables

\begin{tabular}{|c|c|c|c|c|c|}
\hline \multirow[t]{2}{*}{ Variables } & \multicolumn{3}{|c|}{ MUC2 expression } & \multirow[t]{2}{*}{$\chi^{2}$} & \multirow[t]{2}{*}{$\mathrm{P}$-value } \\
\hline & Total & Negative & Positive & & \\
\hline \multicolumn{6}{|l|}{ LN stage } \\
\hline 1 & 889 & $806(61.3)$ & 83 (69.2) & 6.73 & 0.034 \\
\hline 2 & 430 & $406(30.9)$ & $24(20.0)$ & & \\
\hline 3 & 115 & $102(7.8)$ & $13(10.8)$ & & \\
\hline \multicolumn{6}{|l|}{$V I$} \\
\hline No VI & 982 & $882(67.4)$ & $90(77.6)$ & 5.11 & 0.024 \\
\hline Definite & 453 & $427(32.6)$ & $26(22.4)$ & & \\
\hline \multicolumn{6}{|l|}{$E R$} \\
\hline Negative & 435 & 78 (17.9) & $217(49.9)$ & 61.47 & $<0.001$ \\
\hline Positive & 1002 & $60(6.0)$ & $470(46.9)$ & & \\
\hline
\end{tabular}

$\mathrm{LN}=$ lymph node, $\mathrm{VI}=$ vascular invasion.

this as all TMA tumour cores contained only mucinous carcinomas without surrounding normal breast tissue or other in situ lesions.

An inverse association has been identified between those tumours, excluding mucinous carcinomas, expressing MUC2 and nodal spread and vascular invasion. The high level of the gel-forming MUC2 mucin in breast cancer may act as a barrier that interferes with extension of the malignant cells, an advantage that may explain the less aggressive behaviour of mucinous carcinoma of the breast. ${ }^{23}$ We also found a significantly more frequent ERnegative phenotype among MUC2-positive nonmucinous tumours compared with the negative tumours. This can be explained by the relatively higher incidence of grade 3 tumours compared with grade 1 and $2(55.8 \%$ vs 14.4 and $29.85 \%$, respectively), which agrees with a previous study stating that MUC2 overexpression by invasive ductal carcinomas is associated with aggressive tumour behaviour. ${ }^{18,53}$

MUC3 expression appears to have associations with local recurrence and LN stage. However, these results are conflicting as to whether MUC3 expression is, on the whole, a good prognostic indicator or 
not. Local recurrence appears to be more likely with strong MUC3 staining. MUC3 has been shown to be upregulated by steroid hormones in vitro, so aberrant regulation of these compounds in recurring tumours could be the reason for this increased expression. Our results indicate that the expression of membranous MUC3 staining is a potentially poor prognostic feature, with higher grade, poorer NPI and decreased ER expression. Combined with an increase in the potential for vascular invasion, these factors seem to indicate that malignant disease is closely related to membranous MUC3 expression. Therefore, similar to MUC1, the subcellular localization of MUC3 or its altered exppression may also contribute to the process of cancer invasion and metastases. It is possible that MUC3, like MUC1, has roles in cell-cell adhesion, signalling processes or protection from immune reactions and is more capable of increasing the malignancy of a tumour via these mechanisms, something which is harder to achieve within the cell cytoplasm.

Some previous studies have reported that MUC4 may have a prognostic value in cancer including breast cancer. ${ }^{28,29}$ However, we did not find any associations between MUC4 and any of the clinicopathological parameters or patients outcome in our series. The functions that have previously been suggested as possible tumour precipitating factors $^{39,40}$ have not been studied individually in this study, and more work still needs to be carried out to determine the role of this mucin in breast cancer.

In this series, we could not find any association between MUC5AC expression and any of the clinico-pathological variables. Our results correlate with that of Pereira et $a l,{ }^{54}$ who showed that MUC5AC is expressed in a small proportion of tumours and they did not find any associations with prognostic factors in breast cancer.

MUC6 has been associated with many gastrointestinal diseases and is vital in the production of a protective glycoprotein coat to the epithelia of the gut. ${ }^{55,56}$ As well as being linked to gastric carcinomas, MUC6 has been shown to be overexpressed in breast tumours and our results are in concord with Pereira et al, 22 who found positive staining in only 15 out of $65(23.1 \%)$ cases. Analysis of MUC6 expression vs survival showed a potential trend $(P=0.053)$ for MUC6 and patients being alive at the end of the study, as opposed to the weak or negative group. The multivariate analysis of MUC6 compared with grade, stage and size showed MUC6 not to be independent in predicting survival, but it does seem to affect the size of tumour found (the $P$-value of this variable is increased to 0.705), which would normally be a significant factor in determining outcome. The association of MUC6 with survival warrants further study, to determine whether MUC6 could be used as a predictor of breast cancer outcome.

In conclusion, while some mucins (MUC1, MUC3 and MUC4) are expressed in the majority of breast cancer, other mucins (MUC2, MUC5AC and MUC6) are not. Apart from expression of MUC2 and MUC6 in mucinous carcinomas, no association was found between expression of different mucins and tumour type. Among the various mucins expressed in breast cancer, only MUC1 and MUC3 appeared to have the strongest prognostic value in predicting outcome. The presence of MUC1 in the majority of tumour cells is associated with better differentiated tumours and with an improved prognosis. The aberrant expression but not the level of expression of MUC1 may have a prognostic value in predicting patients outcome where the aberrantly localized MUC1 in the tumour cell cytoplasm or nonapical membrane is associated with a shorter survival time. There is little doubt that mucins do have significant roles to play in the development and progression of breast cancer, a fact highlighted by the findings of this study.

\section{References}

1 Fowler J, Vinall L, Swallow D. Polymorphism of the human muc genes. Front Biosci 2001;6:D1207-D1215.

2 Diaz LK, Wiley EL, Morrow M. Expression of epithelial mucins Muc1, Muc2, and Muc3 in ductal carcinoma in situ of the breast. Breast J 2001;7:40-45.

3 Hollingsworth MA, Swanson BJ. Mucins in cancer: protection and control of the cell surface. Nat Rev Cancer 2004;4:45-60.

4 Masaki Y, Oka M, Ogura Y, et al. Sialylated MUC1 mucin expression in normal pancreas, benign pancreatic lesions, and pancreatic ductal adenocarcinoma. Hepatogastroenterology 1999;46:2240-2245.

5 Wesseling J, van der Valk SW, Hilkens J. A mechanism for inhibition of E-cadherin-mediated cell-cell adhesion by the membrane-associated mucin episialin/ MUC1. Mol Biol Cell 1996;7:565-577.

6 Yamamoto M, Bharti A, Li Y, et al. Interaction of the DF3/MUC1 breast carcinoma-associated antigen and beta-catenin in cell adhesion. J Biol Chem 1997;272: 12492-12494.

7 Kondo K, Kohno N, Yokoyama A, et al. Decreased MUC1 expression induces E-cadherin-mediated cell adhesion of breast cancer cell lines. Cancer Res 1998; 58:2014-2019.

8 Yang SZ, Kohno N, Yokoyama A, et al. Decreased E-cadherin augments beta-catenin nuclear localization: studies in breast cancer cell lines. Int J Oncol 2001; 18:541-548.

9 Pandey P, Kharbanda S, Kufe D. Association of the DF3/MUC1 breast cancer antigen with Grb2 and the Sos/Ras exchange protein. Cancer Res 1995;55: 4000-4003.

10 Schroeder JA, Thompson MC, Gardner MM, et al. Transgenic MUC1 interacts with epidermal growth factor receptor and correlates with mitogen-activated protein kinase activation in the mouse mammary gland. J Biol Chem 2001;276:13057-13064.

11 Rahn JJ, Dabbagh L, Pasdar M, et al. The importance of MUC1 cellular localization in patients with breast carcinoma: an immunohistologic study of 71 patients and review of the literature. Cancer 2001;91:1973-1982. 
12 Gendler SJ. MUC1, the renaissance molecule. J Mammary Gland Biol Neoplasia 2001;6:339-353.

13 Gum Jr JR, Hicks JW, Toribara NW, et al. Molecular cloning of human intestinal mucin (MUC2) cDNA. Identification of the amino terminus and overall sequence similarity to prepro-von Willebrand factor. J Biol Chem 1994;269:2440-2446.

14 Yamashita K, Yonezawa S, Tanaka S, et al. Immunohistochemical study of mucin carbohydrates and core proteins in hepatolithiasis and cholangiocarcinoma. Int J Cancer 1993;55:82-91.

15 Zhang S, Zhang HS, Cordon-Cardo C, et al. Selection of tumor antigens as targets for immune attack using immunohistochemistry: protein antigens. Clin Cancer Res 1998;4:2669-2676.

16 Utsunomiya $\mathrm{T}$, Yonezawa $\mathrm{S}$, Sakamoto $\mathrm{H}$, et al. Expression of MUC1 and MUC2 mucins in gastric carcinomas: its relationship with the prognosis of the patients. Clin Cancer Res 1998;4:2605-2614.

17 Clayton F. Pure mucinous carcinomas of breast: morphologic features and prognostic correlates. Hum Pathol 1986;17:34-38.

18 Walsh MD, McGuckin MA, Devine PL, et al. Expression of MUC2 epithelial mucin in breast carcinoma. J Clin Pathol 1993;46:922-925.

19 1Diaz LK, Wiley EL, Morrow M. Expression of epithelial mucins Muc1, Muc2, and Muc3 in ductal carcinoma in situ of the breast. Breast J 2001;7: 40-45.

20 De Bolos C, Garrido M, Real FX. MUC6 apomucin shows a distinct normal tissue distribution that correlates with Lewis antigen expression in the human stomach. Gastroenterology 1995;109:723-734.

21 Ho SB, Shekels LL, Toribara NW, et al. Mucin gene expression in normal, preneoplastic, and neoplastic human gastric epithelium. Cancer Res 1995;55: 2681-2690.

22 Pereira MB, Dias AJ, Reis CA, et al. Immunohistochemical study of the expression of MUC5AC and MUC6 in breast carcinomas and adjacent breast tissues. J Clin Pathol 2001;54:210-213.

23 Matsukita S, Nomoto M, Kitajima S, et al. Expression of mucins (MUC1, MUC2, MUC5AC and MUC6) in mucinous carcinoma of the breast: comparison with invasive ductal carcinoma. Histopathology 2003;42: 26-36.

24 de Bolos C, Guma M, Barranco C, et al. MUC6 expression in breast tissues and cultured cells: abnormal expression in tumors and regulation by steroid hormones. Int J Cancer 1998;77:193-199.

25 Wang RQ, Fang DC. Alterations of MUC1 and MUC3 expression in gastric carcinoma: relevance to patient clinicopathological features. J Clin Pathol 2003;56: 378-384.

26 Carraway KL, Price-Schiavi SA, Komatsu M, et al. Muc4/sialomucin complex in the mammary gland and breast cancer. J Mammary Gland Biol Neoplasia 2001; 6:323-337.

27 Carraway III KL, Rossi EA, Komatsu M, et al. An intramembrane modulator of the ErbB2 receptor tyrosine kinase that potentiates neuregulin signaling. J Biol Chem 1999;274:5263-5266.

28 Price-Schiavi SA, Jepson S, Li P, et al. Rat Muc4 (sialomucin complex) reduces binding of anti-ErbB2 antibodies to tumor cell surfaces, a potential mechanism for herceptin resistance. Int J Cancer 2002;99: 783-791.
29 Nguyen PL, Niehans GA, Cherwitz DL, et al. Membrane-bound (MUC1) and secretory (MUC2, MUC3, and MUC4) mucin gene expression in human lung cancer. Tumour Biol 1996;17:176-192.

$30 \mathrm{Chu}$ JS, Chang KJ. Mucin expression in mucinous carcinoma and other invasive carcinomas of the breast. Cancer Lett 1999;142:121-127.

31 Abd El-Rehim DM, Pinder SE, Paish CE, et al. Expression of luminal and basal cytokeratins in human breast carcinoma. J Pathol 2004;203:661-671.

32 Kew TY, Bell JA, Pinder SE, et al. c-erbB-4 protein expression in human breast cancer. Br J Cancer 2000; 82:1163-1170.

33 Abd El-Rehim DM, Pinder SE, Paish CE, et al. Expression and co-expression of the members of the epidermal growth factor receptor (EGFR) family in invasive breast carcinoma. Br J Cancer 2004;91:1532-1542.

34 Ellis IO, Galea M, Broughton N, et al. Pathological prognostic factors in breast cancer. II. Histological type. Relationship with survival in a large study with long-term follow-up. Histopathology 1992;20:479-489.

35 Elston CW, Ellis IO. Pathological prognostic factors in breast cancer I. The value of histological grade in breast cancer: experience from a large study with longterm follow-up. Histopathology 1991;19:403-410.

36 Galea MH, Blamey RW, Elston CE, et al. The Nottingham Prognostic Index in primary breast cancer. Breast Cancer Res Treat 1992;22:207-219.

37 Kollias J, Murphy CA, Elston CW, et al. The prognosis of small primary breast cancers. Eur J Cancer 1999;35: 908-912.

38 Kononen J, Bubendorf L, Kallioniemi A, et al. Tissue microarrays for high-throughput molecular profiling of tumor specimens. Nat Med 1998;4:844-847.

39 Osako M, Yonezawa S, Siddiki B, et al. Immunohistochemical study of mucin carbohydrates and core proteins in human pancreatic tumors. Cancer 1993; 71:2191-2199.

40 McCarty Jr KS, Miller LS, Cox EB, et al. Estrogen receptor analyses. Correlation of biochemical and immunohistochemical methods using monoclonal antireceptor antibodies. Arch Pathol Lab Med 1985; 109:716-721.

41 Kato N, Endo Y, Tamura G, et al. Mucinous carcinoma of the breast: a multifaceted study with special reference to histogenesis and neuroendocrine differentiation. Pathol Int 1999;49:947-955.

42 Croce MV, Isla-Larrain MT, Rua CE, et al. Patterns of MUC1 tissue expression defined by an anti-MUC1 cytoplasmic tail monoclonal antibody in breast cancer. J Histochem Cytochem 2003;51:781-788.

43 Ellis IO, Bell J, Todd JM, et al. Evaluation of immunoreactivity with monoclonal antibody NCRC 11 in breast carcinoma. Br J Cancer 1987;56:295-299.

44 Baildam AD, Howell A, Barnes DM, et al. The expression of milk fat globule antigens within human mammary tumours: relationship to steroid hormone receptors and response to endocrine treatment. Eur J Cancer Clin Oncol 1989;25:459-467.

45 Muir IM, Reed RG, Stacker SA, et al. The prognostic value of immunoperoxidase staining with monoclonal antibodies NCRC-11 and 3E1.2 in breast cancer. Br J Cancer 1991;64:124-127.

46 Ginestier C, Charafe-Jauffret E, Bertucci F, et al. Distinct and complementary information provided by use of tissue and DNA microarrays in the study of breast tumor markers. Am J Pathol 2002;161:1223-1233. 
47 McGuckin MA, Walsh MD, Hohn BG, et al. Prognostic significance of MUC1 epithelial mucin expression in breast cancer. Hum Pathol 1995;26:432-439.

48 Hayes DF, Mesa-Tejada R, Papsidero LD, et al. Prediction of prognosis in primary breast cancer by detection of a high molecular weight mucin-like antigen using monoclonal antibodies DF3, F36/22, and CU18: a Cancer and Leukemia Group B study. J Clin Oncol 1991;9:1113-1123.

49 Arnerlov C, Ellis IO, Emdin SO. Monoclonal antibody NCRC 11 reactivity with advanced breast carcinoma: lack of prognostic value. Histopathology 1988;13: 695-697.

50 Parham DM, Slidders W, Robertson AJ. Quantitation of human milk fat globule (HMFG1) expression in breast carcinoma and its association with survival. J Clin Pathol 1988;41:875-879.

51 Parham DM, Coghill G, Robertson AJ. Critical evaluation of monoclonal antibody staining in breast carcinoma. J Clin Pathol 1989;42:810-813.
52 O’Connell JT, Shao ZM, Drori E, et al. Altered mucin expression is a field change that accompanies mucinous (colloid) breast carcinoma histogenesis. Hum Pathol 1998;29:1517-1523.

$53 \mathrm{Xu} \mathrm{Y,} \mathrm{Kimura} \mathrm{N,} \mathrm{Yoshida} \mathrm{R,} \mathrm{et} \mathrm{al.} \mathrm{Immunohistochem-}$ ical study of Muc1, Muc2 and human gastric mucin in breast carcinoma: relationship with prognostic factors. Oncol Rep 2001;8:1177-1182.

54 Pereira MB, Dias AJ, Reis CA, et al. Immunohistochemical study of the expression of MUC5AC and MUC6 in breast carcinomas and adjacent breast tissues. J Clin Pathol 2001;54:210-213.

55 Sasaki M, Yamato T, Nakanuma Y, et al. Expression of MUC2, MUC5AC and MUC6 apomucins in carcinoma, dysplasia and non-dysplastic epithelia of the gallbladder. Pathol Int 1999;49:38-44.

56 Reis CA, David L, Correa P, et al. Intestinal metaplasia of human stomach displays distinct patterns of mucin (MUC1, MUC2, MUC5AC, and MUC6) expression. Cancer Res 1999;59:1003-1007. 